ACCEPTED MANUSCRIPT

\title{
Selective activation of memristive interfaces in TaOx-based devices by controlling oxygen vacancies dynamics at the nanoscale
}

To cite this article before publication: CRISTIAN FERREYRA et al 2019 Nanotechnology in press https://doi.org/10.1088/1361-6528/ab6476

\author{
Manuscript version: Accepted Manuscript \\ Accepted Manuscript is "the version of the article accepted for publication including all changes made as a result of the peer review process, \\ and which may also include the addition to the article by IOP Publishing of a header, an article ID, a cover sheet and/or an 'Accepted \\ Manuscript' watermark, but excluding any other editing, typesetting or other changes made by IOP Publishing and/or its licensors" \\ This Accepted Manuscript is @ 2019 IOP Publishing Ltd.
}

During the embargo period (the 12 month period from the publication of the Version of Record of this article), the Accepted Manuscript is fully protected by copyright and cannot be reused or reposted elsewhere.

As the Version of Record of this article is going to be / has been published on a subscription basis, this Accepted Manuscript is available for reuse under a CC BY-NC-ND 3.0 licence after the 12 month embargo period.

After the embargo period, everyone is permitted to use copy and redistribute this article for non-commercial purposes only, provided that they adhere to all the terms of the licence https://creativecommons.org/licences/by-nc-nd/3.0

Although reasonable endeavours have been taken to obtain all necessary permissions from third parties to include their copyrighted content within this article, their full citation and copyright line may not be present in this Accepted Manuscript version. Before using any content from this article, please refer to the Version of Record on IOPscience once published for full citation and copyright details, as permissions will likely be required. All third party content is fully copyright protected, unless specifically stated otherwise in the figure caption in the Version of Record.

View the article online for updates and enhancements. 


\section{Selective activation of memristive interfaces in $\mathrm{TaO}_{x}$-based devices by controlling oxygen vacancies dynamics at the nanoscale}

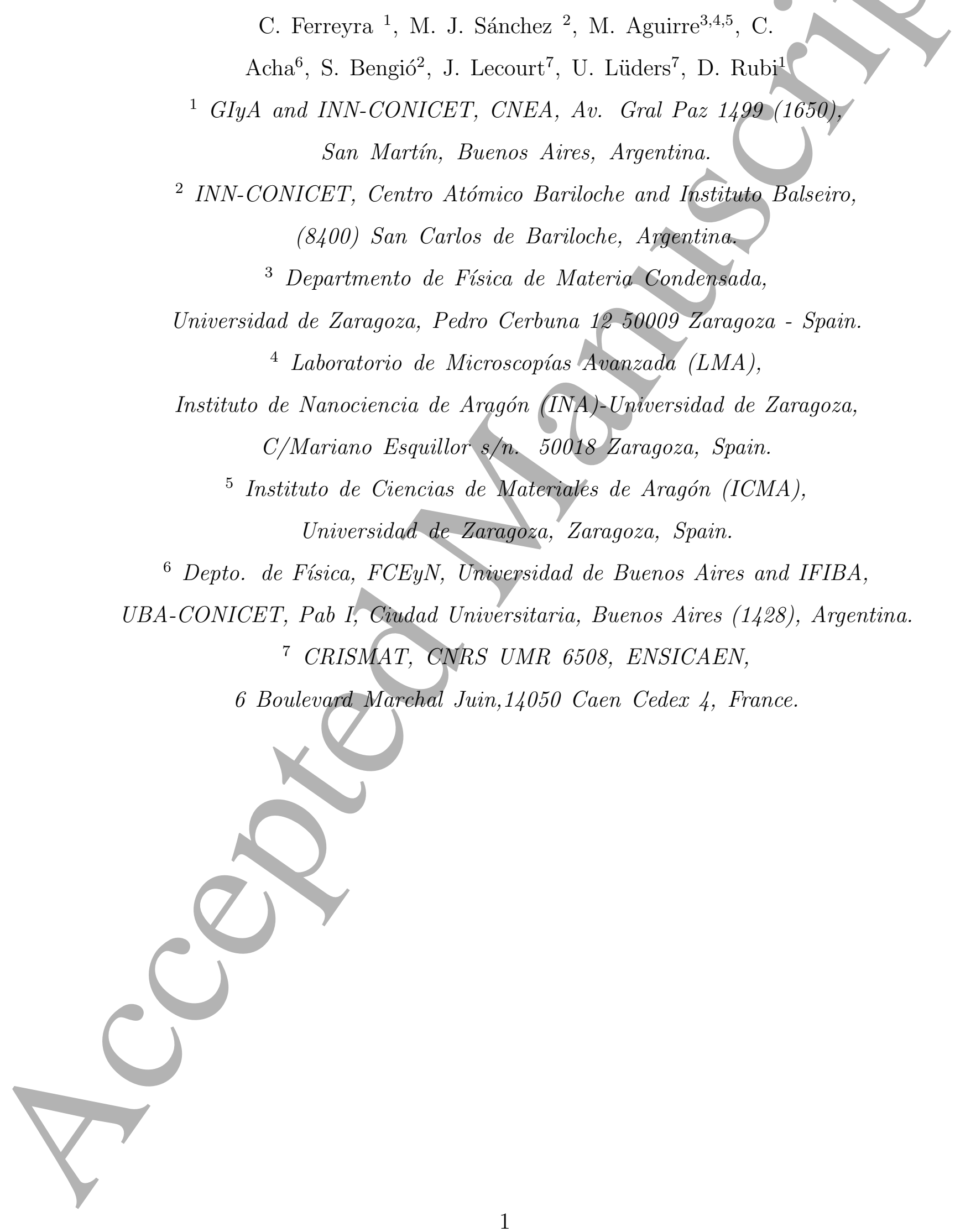


The development of novel devices for neuromorphic computing and non-traditional logic operations largely relies on the fabrication of well controlled memristive systems with functionalities beyond standard bipolar behavior and digital ON-OFF states. In the present work we demonstrate for $\mathrm{Ta}_{2} \mathrm{O}_{5}$-based devices that it is possible to selectively activate/deactivate two series memristive interfaces in order to obtain clockwise or counter-clockwise multilevel squared remanent resistance loops, just by controlling both the electroforming process and the (a)symmetry of the applied stimuli, and independently of the nature of the used metallic electrodes. Based on our thorough characterization, analysis and modeling, we show that the physical origin of this electrical behavior relies on controlled oxygen vacancies electromigration between three different nanoscopic zones of the active $\mathrm{Ta}_{2} \mathrm{O}_{5-x}$ layer: a central one and two quasi-symmetric interfaces with reduced $\mathrm{TaO}_{2-h(y)}$ layers. Our devices fabrication process is rather simple as it implies the room temperature deposition of only one CMOS compatible oxide -Ta-oxide- and one metal, suggesting that it might be possible to take advantage of these properties at low cost and with easy scability. The tunable opposite remanent resistance loops circulations with multiple -analogic- intermediate stable states allows mimicking the adaptable synaptic weight of biological systems and presents potential for non-standard logic devices.

\section{INTRODUCTION}

Non-volatile resistive switching (RS) devices, usually called memristors, are intensively studied due to their potential as resistive random access memories (ReRAM) [1], novel logic units [2] and neuromorphic computing [3] devices. RS has been found for a large number of simple and complex transition metal oxides, including $\mathrm{TaO}_{x}$ [4]. Outstanding memristive figures have been reported in this system, including endurances larger than $10^{12}$ cycles [5], ON-OFF ratios up to $10^{6}$ and retention times greater than 10 years [6]. RS in simple oxides such as $\mathrm{TaO}_{x}$ has been reported as bipolar and the physical mechanism was usually attributed to the modification at the nanoscale of conducting oxygen vacancies (OV) filaments [4]. It is well established that, after forming, the enlargement/retraction of oxygen vacancies filament upon electrical cycling is more favourable in the region close to interfaces presenting an energy barrier (i.e. Schottky barrier formed, for example, when a n-type insulator is in contact with a high work function such as Pt [7]), due to the high local electrical 
field acting at these interfaces. The application of negative voltage to the electrode attracts positively charged oxygen vacancies to the electrode, enlarging the filament and triggering the transition from high resistance (HR) to low resistance (LR). The opposite transition (retraction of the filament) is achieved with positive voltage. Concomitantly, this movement of oxygen vacancies to and from the interface, related to filament enlargement/ryetraction, locally modifies the interface energy barrier [8]. According to the already described SET and RESET polarities, the circulation of the current-voltage (I-V) curve is clockwise in the positive current-positive voltage quadrant, while the remanent resistance vs. voltage curve (usually called Hysteresis Switching Loop, HSL [9]) displays a counter-clockwise behavior.

The possibility of opposite (counter-clockwise) I-V and (clockwise) HSL circulations was also shown for $\mathrm{TiO}_{2} / \mathrm{Pt}$ interfaces, and attributed to oxygen exchange between $\mathrm{Pt}$ and $\mathrm{TiO}_{2}$ at the local position of the filament [8]. Opposite switching polarities were also reported for $\mathrm{TaO}_{x}$ devices and attributed to a competition between ionic motion and electronic i.e charge trapping- effects [10], to the formation of filaments with either conical or hourglass shapes $[11,12]$ or to volumetric oxygen vacancies exchange between $\mathrm{TaO}_{x}$ layers with different stochiometries [13]. The coexistance of two bipolar memristive regimes with opposite polarities in a single device allows to increase or decrease the resistance of the device with stimulus of different amplitudes but the same polarity, which has potential for the development of beyond von Neumann novel computing devices [8].

Asymmetric devices (i.e. an insulating oxide sandwiched between two electrodes of different metals) with an active interface and an ohmic non-active one were reported to display squared HSL $[14,15]$. On the contrary, symmetric systems presenting two similar metal/oxide memristive active interfaces in series were reported to display HSLs with the so-called "table with legs" (TWL) shape $[9,16]$. In these cases, the two interfaces behave in a complementary way [17-19]: when one switches from low resistance (LR) to high resistance (HR), the other switches inversely. Symmetric $\mathrm{Pt} / \mathrm{TiO}_{2} / \mathrm{Pt}$ devices stimulated with symmetric stimuli displayed complex I-V curves with multiple transitions, which was interpreted as bipolar switching taking place simultaneously at both $\mathrm{Pt} / \mathrm{TiO}_{2}$ interfaces [20].

In the present paper we show for $\mathrm{Ta}_{2} \mathrm{O}_{5}$-based devices that the (a)symmetry of the electrical response can be controlled by appropriately tuning the electroforming process and the subsequent excitation protocol, independently of the electrodes symmetry. A careful control of the stimuli protocol allows to selectively activate/deactivate the contribution of two 
memristive interfaces to the overall RS behavior, allowing to obtain both clockwise/counterclockwise multilevel squared HSL or a "table with legs" behavior, where both interfaces are active simultaneously.

From a combination of I-V curves analysis and modeling, togheter with numerical simulations based on the Voltage Enhanced Oxygen Vacancy migration (VEOV) model [9], adapted for binary oxides-based devices [14], we achieve a thorough understanding of the observed phenomenology, which is rationalized in terms of oxygen vacancies electromigration at the nanoscale, between a central zone of the active $\mathrm{Ta}_{2} \mathrm{O}_{5}$ layer and its interfaces with metallic $\mathrm{TaO}_{x}(\mathrm{x}<2)$. Finally, the potential technological implications of the observed tunable opposite HSL circulations with multiple intermediate stable states are discussed.

\section{EXPERIMENTAL SECTION}

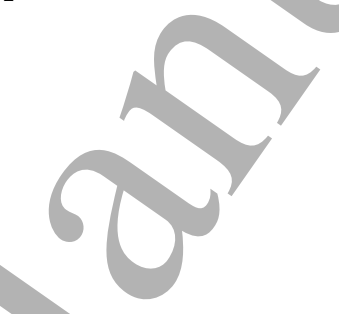

Ta-oxide thin films were deposited on commercial platinized silicon by pulsed laser deposition at room temperature from a single phase $\mathrm{Ta}_{2} \mathrm{O}_{5}$ ceramic target. We used a 2-steps deposition process with oxygen pressures of 0.01 and $0.1 \mathrm{mbar}$, resulting in $35 \mathrm{~nm}$ and $15 \mathrm{~nm}$ layers, respectively, as shown in Figure 1(a). The laser fluence was fixed at $1.5 \mathrm{~J} / \mathrm{cm}^{2}$. For spectroscopic measurements, we also deposited single $\mathrm{TaO}_{x}$ layers at 0.01 and $0.1 \mathrm{mbar}$ of $\mathrm{O}_{2}$, respectively. A Dual Beam Helios 650 was used to acquire scanning electron microscopy images and prepare FIB lamellas to observe cross-sections. High resolution scanning transmission electron microscopy was performed using a FEI Titan G2 microscope at $300 \mathrm{kV}$ with probe corrector and in situ EELS spectrum acquisition with a Gatan Energy Filter Tridiem 866 ERS. The surface composition analysis obtained by X-Ray Photoelectron Spectroscopy (XPS) used a standard Al/Mg twin anode X-ray gun and a hemispherical electrostatic electron energy analyzer. The base chamber pressure was $10^{-9}$ mbar. Top Pt electrodes, 20nm thick, were deposited by sputtering and microstructured by optical lithography. Electrical characterization was performed at room temperature with a Keithley 2612 source-meter connected to a Suss probe station. The bottom Pt electrode was grounded and the electrical stimulus (voltage) was applied to the top Pt electrode. 


\section{RESULTS}

Figure 1(a) shows a STEM-HAADF cross section of a Ta-oxide (bi)layer grown at 0.01 (layer B) and 0.1 (layer A) mbar of $\mathrm{O}_{2}$. Both layers are amorphous and differ in contrast. A brighter image is found for the B layer, indicating a higher relative concentration of Ta (see the blow up displayed in Figure S1 of the Supp. Information), consistently with a more reduced oxide and the lower oxygen pressure used for its growth. Figure 1(b) shows a STEEM-HAADF-EELS map of the bilayer indicating a higher relative concentration of oxygen at the top A layer. Performed quantifications, as the one shown in Figure 1 (c), indicate average oxygen concentrations of $\approx 70 \%$ at and $\approx 60 \%$ at for $\mathrm{A}$ and $\mathrm{B}$ layers, respectively. This suggests average stochiometries for $\mathrm{A}$ and $\mathrm{B}$ layers of $\mathrm{Ta}_{2} \mathrm{O}_{4.70}$ and $\mathrm{TaO}_{1.67}$, indicating in the latter case a mixture of $\approx 67 \% \mathrm{TaO}_{2}$ and $\approx 33 \% \mathrm{TaO}$. Consistent results are obtained from STEEM-HAADF-EDX quantification, as shown in Figure S1 of the Supp. Information.

(a)
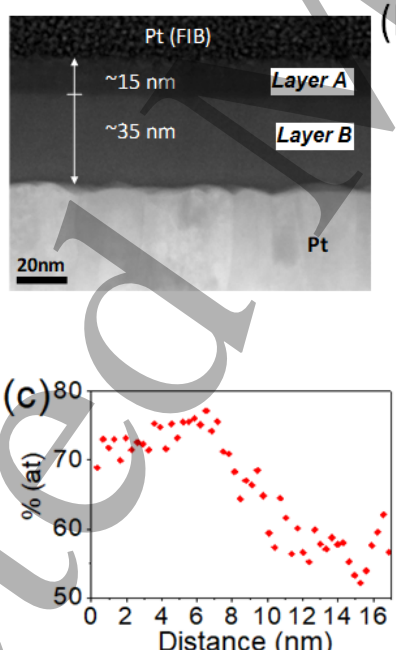

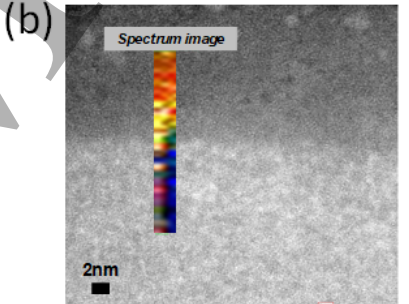

(d)

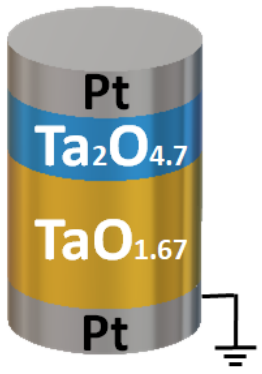

FIG. 1: (a) STEM-HAADF cross-section corresponding to a $\mathrm{TaO}_{x}$ bilayer grown at 0.1 (15nm, layer A) and 0.01 (35nm, layer B) mbar of $\mathrm{O}_{2}$; (b) STEEM-HAADF-EELS line scan (colour map) of oxygen concentration at a zone close to the interface between both layers. The line scan starts in the more oxidized layer and ends in the more reduced one. See text for details; (c) Oxygen concentration quantification from data presented in (c); (d) Sketch showing the geometry and average chemical composition of the pristine devices. The bottom Pt electrode was grounded and electrical stimuli was applied to the top one. 
XPS measurements were performed to obtain information of the films surface chemical composition and Ta oxidation states. Both Ta-4f and $\mathrm{O}-1 s$ spectra are shown in Figure 2 (a) and (b) for $\mathrm{TaO}_{x}$ films grown at 0.1 mbar and 0.01 mbar of $\mathrm{O}_{2}$, respectively. The spectra were fitted using a Voight function for each peak plus a Shirley-type background. The total fitted intensities along with the experimental ones are shown in each spectrum. Ta-4f spectra components are characerized by two identical peaks corresponding to the spinorbit split between $4 \mathrm{f}_{7 / 2}$ and $4 \mathrm{f}_{5 / 2}$ levels, with relative intensities of 3:4. The Ta- $4 \mathrm{f}$ spectrum of the film grown at $0.1 \mathrm{mbar}$ of $\mathrm{O}_{2}$ displays one component corresponding to $\mathrm{Ta}_{2} \mathrm{O}_{5}$ and for the film grown at $0.01 \mathrm{mbar}$ of $\mathrm{O}_{2}$ three components were identified: a major one at binding energy $26.7 \mathrm{eV}$ ascribed to $\mathrm{Ta}_{2} \mathrm{O}_{5}$ and two minor ones at binding energies $25.9 \mathrm{eV}$ and $24 \mathrm{eV}$, ascribed to $\mathrm{TaO}_{2}$ and $\mathrm{TaO}$ respectively. Besides, there is a O-2s component at $27.8 \mathrm{eV}$. The presence of some $\mathrm{Ta}_{2} \mathrm{O}_{5}$ at the spectra of the film grown at low $\mathrm{O}_{2}$ pressure, absent in the case of layer B (buried) in the bilayer analyzed by STEM-HAADF, is related to the ex-situ character of the XPS experiments and indicates surface re-oxidation upon exposure of the film to ambient pressure. It is worth noting that no sputtering process was performed on the films, as it is a possible source of vacancies creation and formation of metastable suboxides [21]. The $\mathrm{O}-1 s$ spectra display a major component at $530.8 \mathrm{eV}$, ascribed to the metal oxide and a minor component at $532.5 \mathrm{eV}$, attributed to adsorbed molecules.

Based on combined STEM-HAADF and XPS analysis, we characterize our virgin devices as $\mathrm{Pt} / \mathrm{Ta}_{2} \mathrm{O}_{4.70} / \mathrm{TaO}_{1.67} / \mathrm{Pt}$, as shown in the sketch displayed on Figure 1(d).

We fabricated devices with different areas, ranging from $\approx 1 x 10^{4} \mu \mathrm{m}^{2}$ to $\approx 70 x 10^{4} \mu \mathrm{m}^{2}$. The devices were initially in a low resistance state, of $\approx 85 \Omega$ for the largest devices and $\approx 350 \Omega$ for the smallest ones. Figure S2 displays the virgin resistance states measured for 3 sets of devices with 6 different areas each. Although it can be observed some variability in the resistance values from device to device for a given area, this variation is low enough to allow a clear visualization of the overall expected scaling of the initial resistance state with the device area (resistance increases as the area diminishes). This indicates a reasonably good device-to-device reproducibility. An electroforming process with negative stimulus (i.e. $-3 \mathrm{~V}$ pulses, with $\mathrm{a} \approx 1 \mathrm{~ms}$ width) was necessary to increase the devices resistance to the range of $\approx k \Omega$ and activate their memristive behaviour. We notice that this electroforming process is different from the typical one found for filamentary memristive behavior, where the pristine high resistance state drops to a lower resistance one upon forming. A simi- 
lar electroforming process than ours was found, for instance, for area-related memristive behavior in $\mathrm{Ti} /$ manganite devices [22].

After forming, room temperature dynamic current-voltage (I-V) curves were obtained by applying a sequence of voltage pulses of different amplitudes $\left(0 \rightarrow V_{\max } \rightarrow-V_{\min } \rightarrow 0\right.$, with a time-width of $1 \mathrm{~ms}$ and a step of $100 \mathrm{mV}$ ), with the current measured during the application of the pulse. Additionally, after each writing voltage pulse a small undisturbing voltage of $100 \mathrm{mV}$ was applied to extract the remanent resistance (the current I was measured and the remanent resistance state was calculated as $\mathrm{R}_{R E M}=0.1 \mathrm{~V} / \mathrm{I}$ ), in order to obtain the corresponding HSL. We have found that the devices electrical response is highly dependant on both $V_{\max }$ and $V_{\min }$.

Figures 3 (a) and 4 (a) display, respectively, the dynamic I-V curve and HSL obtained for a device with $28 \times 10^{3} \mu \mathrm{m}^{2}$ area, for $V_{\max }=+1.5 \mathrm{~V}$ and $V_{\min }=-2 \mathrm{~V}$. It is found that the transition from $\mathrm{HR}_{2}$ to $\mathrm{LR}_{2}$ (SET process, the notation of the resistive states is chosen to be consistent with the fittings and simulations to be described below) is achieved with positive stimulus $\left(V_{S} \approx+1 \mathrm{~V}\right)$ while the opposite transition (RESET) is obtained with negative 

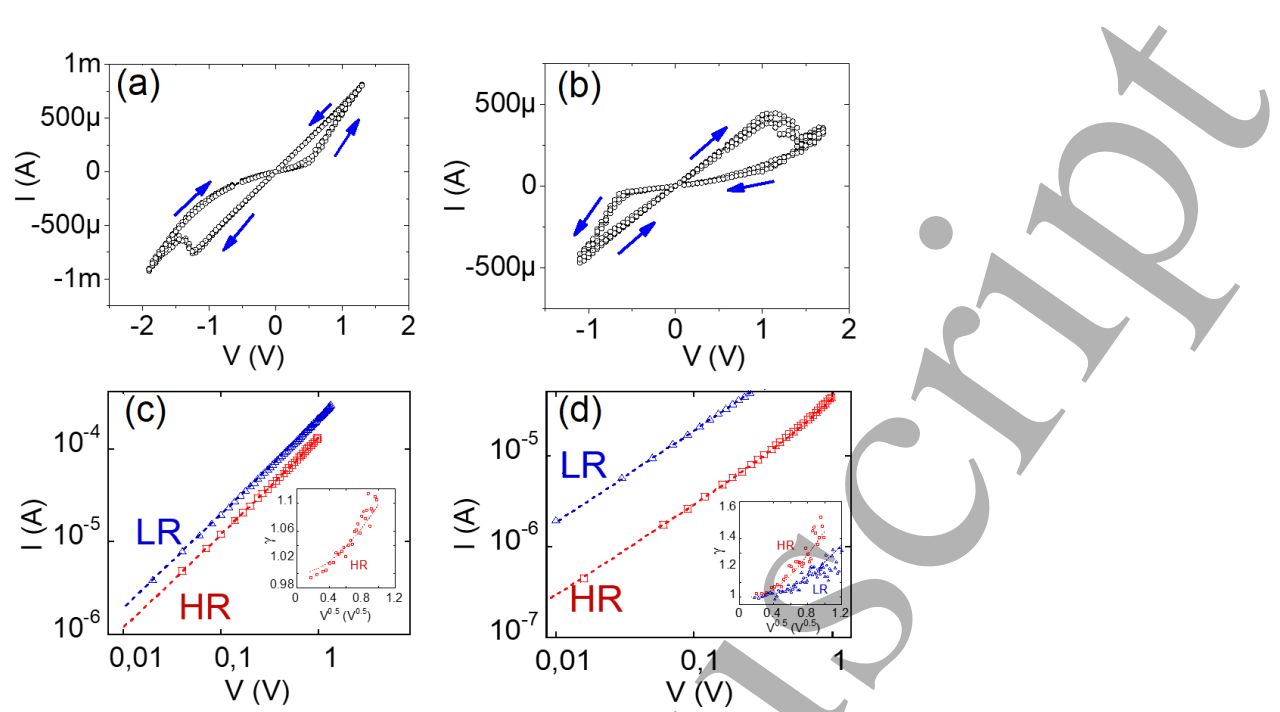

FIG. 3: I-V response of a device with $28 \times 10^{3} \mu \mathrm{m}^{2}$ area, obtained for voltage excursions between $V_{\max }=+1.5 \mathrm{~V}$ and $V_{\min }=-2 \mathrm{~V}(\mathrm{a})$, and for $V_{\max }=2 \mathrm{~V}$ and $V_{\min }=-1.5 \mathrm{~V}(\mathrm{~b})$, where the circulation of the I-V curve is inverted. We notice that (a) and (b) corrrespond to the same device. (c),(d) Fittings -in dashed lines- performed on I-V curves recorded for a $6.4 \times 10^{3} \mu \mathrm{m}^{2}$ device, for the high and low resistance states, labeled respectively $\mathrm{HR}$ and LR. The used equivalent circuit is described in the text and Supp. Information. The insets show the associated $\gamma$ vs $\mathrm{V}^{1 / 2}$ representation for the LR ans HR. For better visualization, the LR state was not included in the inset of panel (c). The dashed line corresponds to the theoretical $\gamma$ curve, extracted from the I-V curves fittings.

voltage $\left(V_{R} \approx-1.2 \mathrm{~V}\right)$. The $\mathrm{LR}_{2}$ and $\mathrm{HR}_{2}$ states are $0.8 \mathrm{k} \Omega$ and $2.5 \mathrm{k} \Omega$ respectively, giving an $\mathrm{ON}-\mathrm{OFF}$ ratio of 3.1 . The stability and reproducibility of the curves is remarkable, as shown in Figure S3 of the Suppl. Information for 200 consecutive cycles. In addition, retention times up to $10^{4} \mathrm{~s}$ were checked for both resistive states.

From these measurements, it is established that the HSL present a clockwise (CW) circulation. Remarkably, this circulation is inverted to a counter-clockwise (CCW) behaviour when the maximum voltage excursion are inverted to $V_{\max }=+2 \mathrm{~V}$ and $V_{\min }=-1.5 \mathrm{~V}$, as shown in Figures 3 (b) and 4 (d), respectively. In this case, the transition from $\mathrm{HR}_{1}$ to $\mathrm{LR}_{1}$ (SET process) is achieved with negative stimulus $\left(V_{S} \approx-1 \mathrm{~V}\right)$ while the opposite transition (RESET) is obtained with positive voltage $\left(V_{R} \approx+1.5 \mathrm{~V}\right)$. The $\mathrm{LR}_{1}$ and $\mathrm{HR}_{1}$ states in this case are $1 \mathrm{k} \Omega$ and $3.3 \mathrm{k} \Omega$ respectively, giving an ON-OFF ratio of 3.3. Again, an excellent stability upon consecutive cycling is found (200 cycles), as shown in Fig. S3 of the Supp. 
Information, and retention times for up $10^{4} \mathrm{~s}$ were also checked. These results clearly show that the circulation of the HSL can be controlled and tuned on the same device by properly choosing the voltage excursions of applied stimuli. The inverse dependance of $\mathrm{LR}_{1}$ and $\mathrm{LR}_{2}$ states with the device area (shown in Figure S4 of the Supp. Information) indicates the existance of conducting paths comprising the complete device area.

Interestingly, if the voltage excursions are enlarged and symmetrized to $V_{\max }=-V_{\min }=2$ $\mathrm{V}$, the HSL change from squared to a TWL-like shape, as shown in Figure 4 (c). The squared HSLs of Figures 4 (a) and (b) correspond to a single active interface while the TWL of Figure 4 (c) corresponds to two complementary active interfaces; that is, when one switches from low to high resistance the other one changes inversely [9]. These results clearly indicate that our two memristive series interfaces can be selectively decoupled and activated by means of the application of proper electrical stimuli, and goes against previous claims about the "simultaneous" memristive behavior of two series interfaces [20].

We have found that for both (squared) HSL circulations multilevel states are possible. Figure 5 (a) shows, for a device with $11.3 \times 10^{3} \mu \mathrm{m}^{2}$ area, that different non-volatile resistance levels can be achieved for CW HSLs upon increasing $V_{S}$ from 1.3 to $1.9 \mathrm{~V}$, while keeping $V_{R}$ fixed at $-1.9 \mathrm{~V}$. In a similar way, Figure 5 (b) shows a multi-level behavior for a CCW HSL upon changing $V_{S}$ from $-1.3 \mathrm{~V}$ to $-1.9 \mathrm{~N}$ while keeping $V_{R}=1.2 \mathrm{~V}$ fixed. We note that this device presents higher remanent resistance states due to its lower area in relation to the previously described devices of Figures 3 and 4.

The multilevel resistance states show that our devices behave in an analogic way, which is a key feature to mimic the adaptable sinaptic weight of biological synapsis. In our case, the knob that allows this behavior is the RESET voltage, in opposition to protocols that use the compliance current to obtain multilevel storage [23].

Our devices switching poyer can be estimated from the RESET events of Figures 4(a) and (b), corresponding to a $95 \mu \mathrm{m}$ device, by using the expression $\mathrm{P}=\mathrm{V}^{2} / \mathrm{R}_{R E M}$. A switching power of $\approx 10 \mathrm{~mW}$ is obtained, which is in perfect agreement with the scaling law between RRAMs switching power and device size reported by Yang et al. [24]. Considering that our RESET transitions are completed after the application of $\approx 10$ pulses of $\approx 1$ ms timewidth, a switching energy of $\approx 10 \mu \mathrm{J}$ is estimated. This energy value is also comparable to reported values for $\mathrm{HfO}_{x} / \mathrm{TiO}_{x}$ devices with similar size than ours [25]. Also, a comparison with existing FLASH technology is possible. For instance, 45nm NOR-FLASH memories 
consume about $100 \mathrm{pJ} / \mathrm{bit}$; in order to obtain a meaningful comparison between the latter value and our devices switching energy, it is necessary to extrapolate our devices switching energy from their $95 \mu \mathrm{m}$ size to the $45 \mathrm{~nm}$ limit, by using the scaling law between RRAMs switching power and device size reported by Yang et al. [24]. This gives for our system an estimated $45 \mathrm{~nm}$-switching energy of $\approx 50 \mathrm{pJ}$, which is comparable to the already mentioned figure of $45 \mathrm{~nm}$ NOR-FLASH memories.
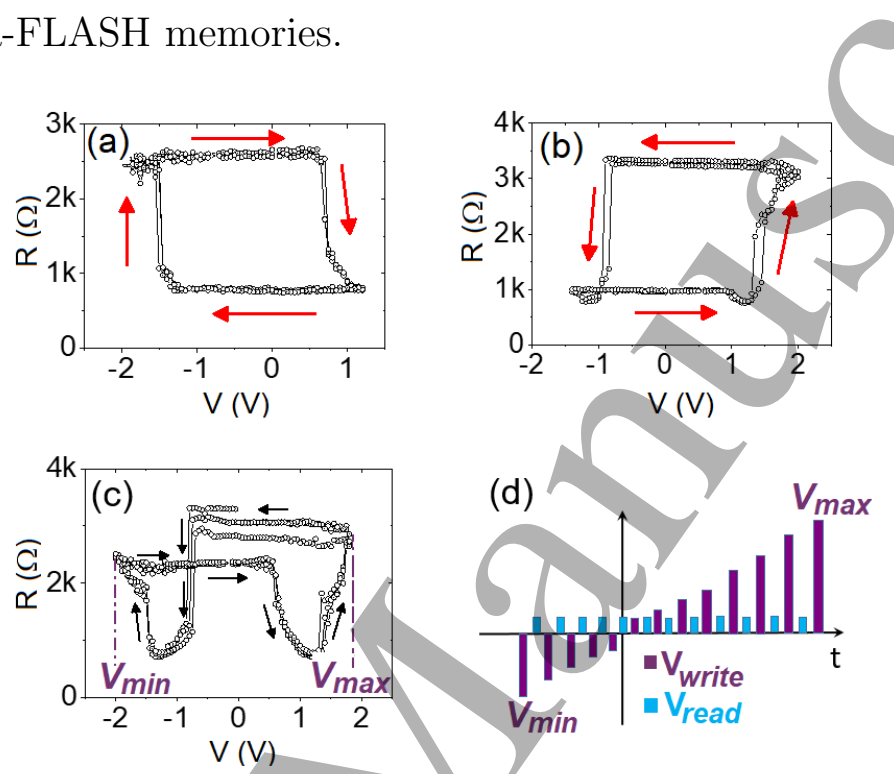

(d)
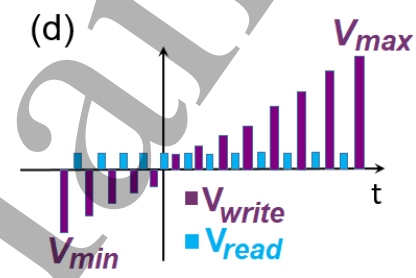

FIG. 4: HSL's with (a) CW circulation and (b) CCW circulation, for asymmetric voltage excursions; (c) TWL HSL obtained for symmetric voltage excursions, showing two complementary active interfaces. The sâme device than in Figures 3 (a) and (b) was used;

(d) Voltage protocol used to obtain dynamic I-V curves and HSL. $V_{\max }\left(V_{\min }\right)$ is the maximum (minimum) voltage excursion for the write pulses. Notice the different values of $V_{\text {min }}$ and $V_{\text {max }}$ for the CW and CCW HSL's of panels (a) y (b), respectively.

In order to understand the observed memristive behavior, we start with the forming process. Virgin devices are found in a low resistance state, indicating that the more oxidated A layer (with an average stochiometry $\mathrm{Ta}_{2} \mathrm{O}_{4.70}$, according to STEEM-HAADF experiments) presents a substantial higher conductivity that the one expected for the insulating stochiometric Ta-pentoxide. This can be explained both by the presence of strong oxygen deficiency and to some material inhomogeneity at the nanoscale (see Figure S1 of the Supp. Information), which allow the presence of percolative low resistance Ta-rich paths bridging the nearby layers. However, as both $\mathrm{TaO}_{2}$ and $\mathrm{TaO}$ present at the $\mathrm{B}$ layer are metallic [4], the A layer still dominates the resistance of the oxide bilayer. The application of negative voltage 
to the top electrode (left in Figure 6 (a)) triggers an electroforming process that increases the resistance of the device by inducing the migration of OVs within the A layer, as the electrical field acting on this layer is higher that the one acting on the more reduced $\mathrm{B}$ layer. Oxygen ions are pushed down within the A layer (from left to right in the scheme of Figure 6 (a)), leading to the formation of a nearly stochiometric $\mathrm{Ta}_{2} \mathrm{O}_{5-x}$, resistive, layer and a strongly reduced, metallic, layer in contact with the Pt top (left zone in Figure 6 (a)) eléctrode. Unlike the case of Ti-oxides, which display a large number of stable sub-stochiometric phases with less oxygen content than $\mathrm{TiO}_{2}$ [26], the commonly accepted Ta-oxide phase diagram only allows stable $\mathrm{Ta}_{2} \mathrm{O}_{5}$ and $\mathrm{Ta}(\mathrm{O})$-that is metallic Ta with some diluted oxygen- phases [27]. $\mathrm{TaO}_{2}$ has been considered as a metastable phase [28], but recent reports claim that it might be stable with an even lower energy than $\mathrm{Ta}_{2} \mathrm{O}_{5}[29,30]$. This suggests that the as-grown A layer, with nominal $\mathrm{Ta}_{2} \mathrm{O}_{4.70}$ composition, might be a metastable phase that is phase separated during forming, due to Joule heating [28], into nearly stochiometric stable $\mathrm{Ta}_{2} \mathrm{O}_{5-x}$ and $\mathrm{TaO}_{2-h}$ layers $(x, h<<1$ ), as depicted in Figure 6 (a). If we assume that the forming process only involves oxygen redistribution within the A layer, it can be estimated that the thicknesses of $\mathrm{Ta}_{2} \mathrm{O}_{5-x}$ and $\mathrm{TaO}_{2-h}$ layers after forming are about $90 \%$ and $10 \%$ of the initial A layer thickness $(\approx 15 \mathrm{~nm})$.

In consequence, for negative electroforming, our post-forming stack is $\mathrm{Pt} / \mathrm{TaO}_{2-h} / \mathrm{Ta}_{2} \mathrm{O}_{5-x} / \mathrm{TaO}_{2-y} / \mathrm{Pt}$. The absence of $\mathrm{Pt} / \mathrm{Ta}_{2} \mathrm{O}_{5-x}$ interfaces, which are known to be Schottky and rectifying [5, 31], is confirmed by the symmetric and non-rectifying behavior observed in the post-forming I-V curves (Figures 3 (a) and (b)). Interestingly, if the electroforming polarity is inverted to positive voltage (see Figure S5 in the Suppl. Information), leading to a post-forming $\mathrm{Pt} / \mathrm{Ta}_{2} \mathrm{O}_{5-x} / \mathrm{TaO}_{2-h} / \mathrm{TaO}_{2-y} / \mathrm{Pt}$ stack, the $\mathrm{Ta}_{2} \mathrm{O}_{5-x}$ layer is formed now in contact with Pt, producing the diode-like behaviour shown in Figure S5.

We also remark that after negative electroforming the $\mathrm{Ta}_{2} \mathrm{O}_{5-x}$ resistive layer is sandwiched between two reduced metallic $\mathrm{TaO}_{2-h}$ and $\mathrm{TaO}_{2-y}$ layers, giving a quasi-symmetric geometry, as shown in the zoomed sketch of Figure 6 (b), which explains the HSL with TWL-like shape obtained for symmetric stimuli (Figure 4 (c)), typical of symmetric devices.

The scenario proposed above is supported by the following analysis and fittings performed on the room temperature post-forming I-V curves, considering the $\gamma=\mathrm{d} \operatorname{Ln}(\mathrm{I}) / \mathrm{d} \operatorname{Ln}(\mathrm{V})$ parameter representation [32]. This method proved to be useful in order to reveal the presence of a mixture of conduction mechanisms [33, 34], as usually found in oxide-based 

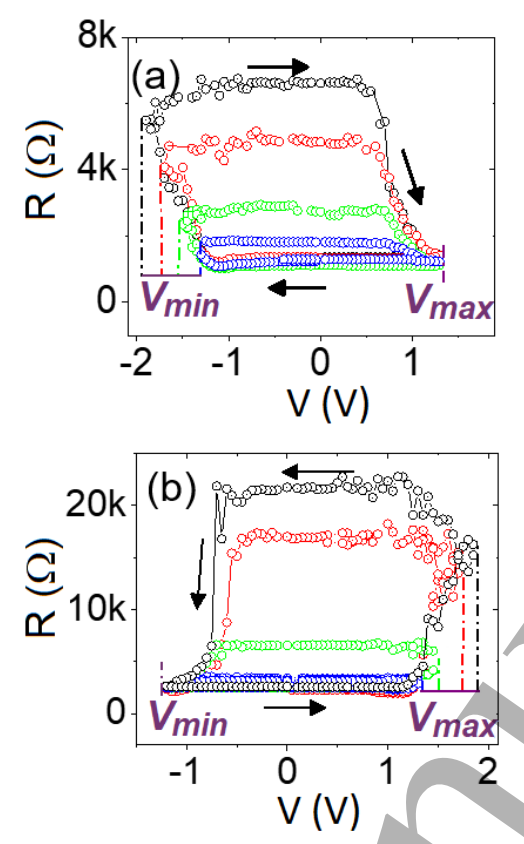

FIG. 5: Multilevel HSL recorded on a device with $11,3 \times 10^{3} \mu \mathrm{m}^{2}$ area for (a) CW circulation and (b) CCW circulation. In (a) $\mathrm{V}_{\max }$ is fixed while $\mathrm{V}_{\min }$ is changed; in (b) $\mathrm{V}_{\min }$ is fixed while $\mathrm{V}_{\max }$ is changed.

devices [35-37]. As can be observed in the insets of Figures 3 (c) and (d), associated with both CCW and CW HSLs of devices with $6.4 \times 10^{3} \mu \mathrm{m}^{2}$ area, the dependence of $\gamma$ vs $\mathrm{V}^{1 / 2}$ indicates the existence of an ohmic conduction $(\gamma \simeq 1$ at low voltages) in parallel with a mild non-linear space charge limited current (SCLC) conduction ( $\gamma$ increases smoothly with $\mathrm{V}$ and remains $<2$ ).

The simplest schematic circuit representation derived from the voltage dependence of the $\gamma$ parameter consists in two parallel SCLC-ohmic conducting channels in series, as sketched in Figures 6(c) and (d), for CCW and CW HSLs, respectively. The experimental I-V curves were fitted by considering the equivalent circuit and the corresponding equations, as described in the Supp. Information. The performed fits give an excellent reproduction of the experimental I- $V$ characteristics as well as the associated $\gamma$ vs. $\mathrm{V}^{1 / 2}$ curves, as shown in Figures 3 (c) and (d). We notice that no good fitting was obtained if Schottky emission is assumed for the non-linear element of the equivalent circuit. This fact, together with the already discussed non-rectifying behavior observed in the I-V curves (Figures 3 (a) and (b)) rule out the presence of $\mathrm{Ta}_{2} \mathrm{O}_{5-x}$ in contact with the left Pt electrode, giving consistency to the proposed post-forming scenario. 
(a)

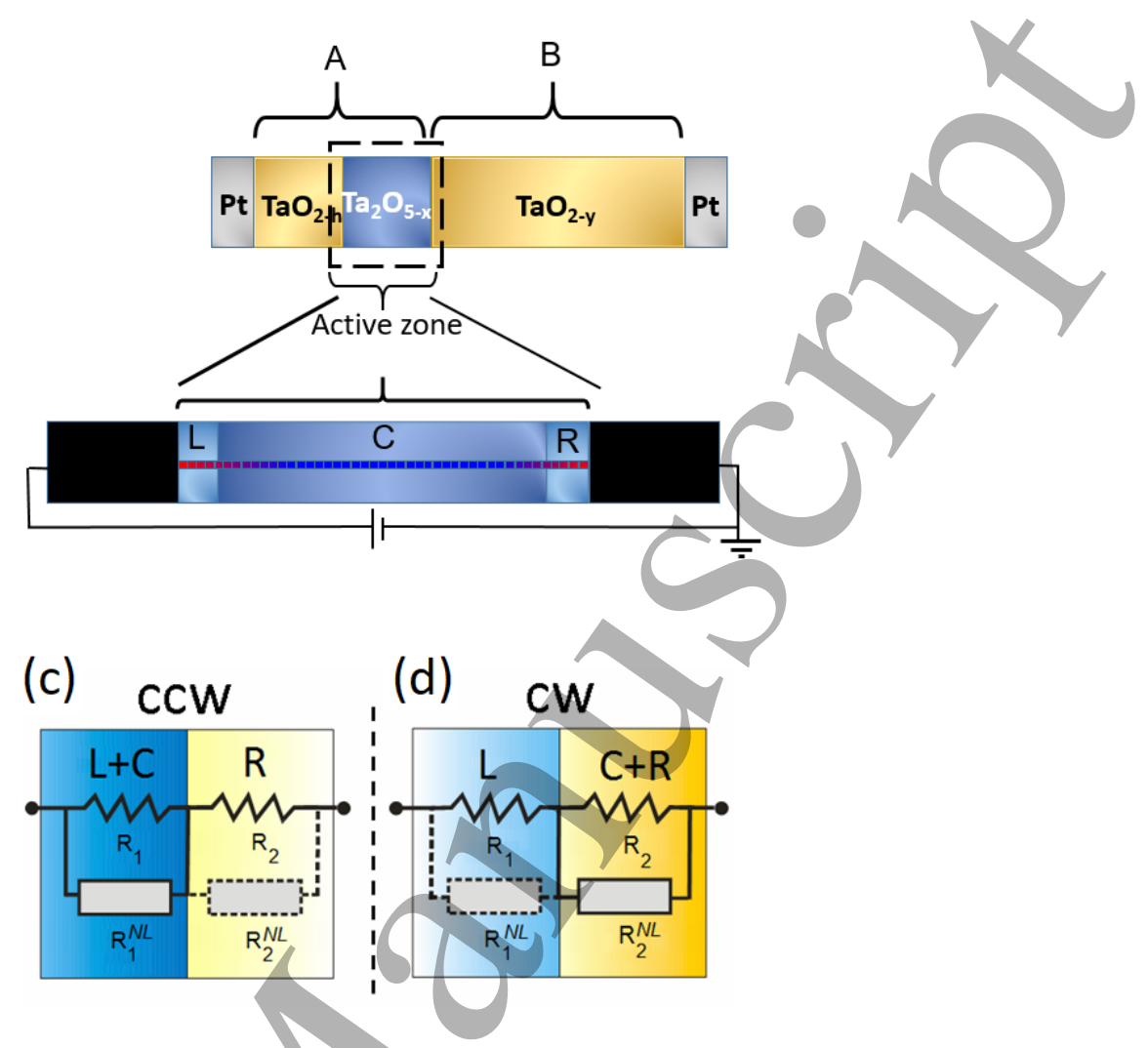

(b)

FIG. 6: (a) Schematic diagram of the post-formed device. The initial asymmetric geometry turns into a quasi-symmetric $\mathrm{Pt} / \mathrm{TaO}_{2-h} / \mathrm{Ta}_{2} \mathrm{O}_{5-x} / \mathrm{TaO}_{2-y} / \mathrm{Pt}$ stack after forming; (b) Sketch of the post-forming active region for the memristive behavior and scheme of the 1D chain of nanodomains assumed for the numerical simulations with the VEOV model. See text for details; (c), (d) Equivalent circuit model that describes CCW HSL and CW HSLs, respectively. $\mathrm{R}_{i}$ indicate ohmic resistors, while $\mathrm{R}_{i}^{N L}$ non-linear SCLC elements. $\mathrm{L}$ and $\mathrm{R}$ represent $\mathrm{TaO}_{2-h} / \mathrm{Ta}_{2} \mathrm{O}_{5-x}$ and $\mathrm{Ta}_{2} \mathrm{O}_{5-x} / \mathrm{TaO}_{2-y}$ interfaces, while $\mathrm{C}$ represents the bulk central $\mathrm{Ta}_{2} \mathrm{O}_{5-x}$ zone in between. The dashed-lines indicate that SCLC-non-linear element is short-circuited by the low resistance ohmic resistor in parallel.

The memristive and non-linear behaviours are nicely reflected in the extracted values of the equivalent circuit elements, listed in Table I. As can be observed, the electric response associated with the CCW HSL is dominated by $\mathrm{R}_{1}$ and $\mathrm{R}_{1}^{N L}$ elements, as the $\mathrm{R}_{2}$ resistor is highly metallized, shortcircuiting the $\mathrm{R}_{2}^{N L}$ non-linear element. The equivalent resistance of the circuit $\mathrm{R}_{1}^{e q}$ is basically the parallel combination between $\mathrm{R}_{1}$ and $\mathrm{R}_{1}^{N L}$. When the electric response switches to the $\mathrm{CW} H \mathrm{HLL}$, the situation is reversed: $\mathrm{R}_{1}$ shortcircuits the non-linear $\mathrm{R}_{1}^{N L}$ element and the resistance of the device is dominated by the parallel combination 
TABLE I: Values in $\mathrm{k} \Omega$ of the equivalent-circuit elements obtained by fitting the experimental I-V characteristics with the corresponding equations (see Supp. Information). Values at $0.1 \mathrm{~V}$ and $1 \mathrm{~V}$ are indicated for the non-linear SCLCelement.

\begin{tabular}{|c|c|c|c|c|c|c|c|c|}
\hline \hline $\mathbf{H S L}$ & $\mathbf{S T A T E}$ & $\mathbf{R}_{1}$ & $\mathbf{R}_{1}^{N L} \mathbf{( 0 . 1 V )}$ & $\mathbf{R}_{1}^{N L} \mathbf{( 1 \mathbf { V } )}$ & $\mathbf{R}_{2}$ & $\mathbf{R}_{2}^{N L} \mathbf{( 0 . 1 V )}$ & $\mathbf{R}_{2}^{N L} \mathbf{( 1 \mathbf { V } )}$ & $\mathbf{R}_{R E M}$ \\
\hline \hline $\mathbf{C C W}$ & HR & 34.2 & 1700 & 70 & $\leq 0.8$ & - & - & 34.3 \\
& LR & 5.2 & 620 & 25 & $\leq 0.1$ & - & - & 5.3 \\
\hline \multirow{2}{*}{$\mathbf{C W}$} & HR & $\leq 0.1$ & - & - & 8.4 & 770 & 8 & 8.4 \\
& LR & $\leq 0.1$ & - & - & 5.1 & 500 & 50 & 5.1 \\
\hline \hline
\end{tabular}

between $\mathrm{R}_{2}$ and the non-linear $\mathrm{R}_{2}^{N L}$ element. We notice that at low voltage and current values the non-linear elements present a much higher resistance than the parallel ohmic resistors, and therefore the equivalent resistance is mainly determined by the latter. As displayed by Table I, the equivalent resistances at $100 \mathrm{mV}$ are basically $\mathrm{R}_{1}$ (CCW HSL) and $\mathrm{R}_{2}$ (CW HSL), respectively, which perfectly match the experimental remanent values $\mathrm{R}_{R E M}$

Further support to the proposed equivalent circuit and transport mechanisms can be obtained from temperature electric measurements, as reported, for example, by Long et al. [25]. For that, we characterized the electrical response of our system at temperatures between $\mathrm{RT}$ and $\approx 400 \mathrm{~K}$. The evolution of the remanent resistance with temperature for both high and low resistance states corresponding to CW HSL, shown in Figure S7, display a linear increase, typical of metallic elements, with temperature coefficients $\alpha=0.011$ 1/K and $\alpha^{\prime}=0.0021 / \mathrm{K}$ for $\mathrm{HR}$ and $\mathrm{LR}$ states, respectively. As expected for the low voltage stimulus used to measure the remanent resistance, the parallel resistor $R_{2}$ of the equivalent circuit (displaying a much lower resistance than the parallel non-linear element at $100 \mathrm{mV}$, see Figures 6(c) and (d)) dominates the temperature dependence of the electrical properties, indicating its metallic nature for both states. A similar analysis can be done for the $\mathrm{CCW}$ remanent resistance states. The influence of the non-linear element, present in the equivalent circuit (see Figures 6(c) and (d)), on the temperature electrical properties is more subtle, and can be determined by analysing and fitting the I-V curves at different temperatures, as done in the Suppl. Info. The performed analysis, shown in Figures S8 and S9 and described in detail in the Suppl. Info., confirms the SCLC nature of the non-linear element included in our equivalent circuit. 
The physical origin of the observed memristive behavior can be understood in terms of OVs electromigration between a central zone of the $\mathrm{Ta}_{2} \mathrm{O}_{5-x}$ layer and the interfacial zones close to $\mathrm{TaO}_{2-h}$ and $\mathrm{Ta}_{2-y}$ layers, respectively. To achieve a behavior consistent with the migration of OVs induced by the electric field, the CCW HSL should be represented by an active SCLC-ohmic zone, represented by the parallel $\mathrm{R}_{1}$ and $\mathrm{R}_{1}^{N L}$ circuit elements, which includes the left interface with $\mathrm{TaO}_{2-h}$ plus the central $\mathrm{Ta}_{2} \mathrm{O}_{5-x}$ zone (we recall that SCLC is a bulk conduction mechanism) where the memristive effects esentially modify this sector, while the ohmic element $\mathrm{R}_{2}$ represents the right interface with $\mathrm{TaO}_{2-y}$, characterized by a rather low resistance. On the contrary, for the CW HSL, the increase of the amplitude of the negative applied pulses switches the active element to a zone comprising the right interface with $\mathrm{TaO}_{2-y}$ plus the central zone, both represented by $\mathrm{R}_{2}$ and $\mathrm{R}_{2}^{N L}$, increasing its resistance and concomitantly decreasing the resistance of the $\mathbb{R}_{1}$ ohmic element, which now short-circuits the SCLC $\mathrm{R}_{1}^{N L}$ element (see Figures 6 (c) and (d) for sketchs depicting these situations).

In the next section we will carefully address by means of simulations the OV dynamics linked to this behavior.

\section{NUMERICAL SIMULATIONS}

Based on the previous description, we analize here the electrical response obtained with the voltage enhanced oxygen vacancies drift (VEOV) model, which allows simulating OV dynamics at the nanoscale, linked to the memristive behavior of our devices. We start by reviewing the main equations, and we refer the readers to References 9 and 14, for further details.

We consider that the active memristive region is the $\mathrm{Ta}_{2} \mathrm{O}_{5-x}$ layer, which is assumed to consist of three zones: a central one labelled as $\mathrm{C}$ and two zones, left (L) and right (R), localized close to the interfaces with the metallic $\mathrm{TaO}_{2-h(y)}$ layers, which present different properties due to the presence of interfacial disorder or defects. A sketch of the assumed memristive active zone is shown in Figure 6 (b). For the modelling we suppose a 1D chain of $N=N L+N C+N R$ total sites. The first $N L$ sites correspond to the L layer, the following $N C$ sites are assigned to the central $\mathrm{C}$ layer and the last $N R$ sites are linked to the $\mathrm{R}$ layer and we assume $N C>N L=N R$. The sites, characterized by their resisitivity, physically 
represent small domains of (sub)nanoscopic dimensions with an initial OVs concentration that we assume correspond to the post forming state.

An universal feature of oxides is that their resistivity is affected by the precise oxygen stoichiometry. In particular, $\mathrm{Ta}_{2} \mathrm{O}_{5}$ behaves as an n-type semiconductor in which oxygen vacancies reduce its resistivity. Therefore, we write the resistivity $\rho_{i}$ of each site $i$ as a linear decrescent (most simple) function of the local OV density $\delta_{i}$, namely:

$$
\rho_{i}=\rho_{0}\left(1-A_{i} \delta_{i}\right)
$$

where $\rho_{0}$ is the residual resistivity for negligible OV concentration $\left(\delta_{i}=0\right)$ and $\mathrm{A}_{i}$ is a factor that changes between $\mathrm{C}, \mathrm{L}$ and $\mathrm{R}$ layers, satisfying $A_{i} \delta_{i}<1 \forall i$. We consider $\mathrm{A}_{L}, \mathrm{~A}_{R}<$ $\mathrm{A}_{C}$, which implies that the resistivity of the interfacial zones is less sensitive to the presence of $\mathrm{OV}$ due to the presence of disorder or defects. Also, the coefficients $A_{i}$ can be taken for each layer either smoothly dependent on the site position or as constants (as we do for simplicity), without affecting the qualitative behaviour of the simulated results [38].

Following Equation(1), the total resistivity of the system is given by:

$$
\rho \equiv \rho_{s}-\rho_{0}\left(\sum_{i=1}^{N L} A_{L} \delta_{i}-\sum_{i=N L+1}^{N-N R} A_{C} \delta_{i}-\sum_{i=N-N R+1}^{N} A_{R} \delta_{i}\right),
$$

being $\rho_{s} \equiv N \rho_{0}$.

Given an external voltage $V(t)$ applied at time $t$, the OV density at site $i$ is updated for each simulation step according to the rate probability $p_{i j}=\delta_{i}\left(1-\delta_{j}\right) \exp \left(-V_{\alpha}+\Delta V_{i}\right)$, for a transfer from site $i$ to a nearest neighbor $j=i \pm 1$. Notice that $p_{i j}$ is proportional to the OV density present at site $i$, and to the available concentration at the neighbour site $j$. In order to restrict the dynamics of OVs to the active region, we take $p_{01}=p_{10}=p_{N N+1}=p_{N+1 N}=0$. In addition as the total density of vacancies is conserved, for each simulation step it is satisfied that $\sum_{i=1}^{N} \delta_{i}=N \delta_{0}$, being $\delta_{0}$ the OV density per site for an uniform distribution (assumed as known, see Supp. Information section).

In the Arrhenius factor, $\exp \left(-V_{\alpha}+\Delta V_{i}\right), \Delta V_{i}$ is the local potential drop at site $i$ defined as $\Delta V_{i}(t)=V_{i}(t)-V_{i-1}(t)$ with $V_{i}(t)=V(t) \rho_{i} / \rho$ and $V_{\alpha}$ the activation energy for vacancy diffusion in the absence of external stimulus. We consider values of $V_{\alpha}=V_{L}, V_{C}$ and $V_{R}$ for the $\mathrm{L}, \mathrm{C}$ and $\mathrm{R}$ layers respectively. All the energy scales are taken in units of the thermal energy $k_{B} T[38]$. 
According to standard RS experiments, we chose the stimulus $V(t)$ as a linear ramp following the cycle $0 \rightarrow V_{m 1} \rightarrow-V_{m 2} \rightarrow 0$. At each simulation time step $t_{k}$ we compute the local voltage profile $V_{i}\left(t_{k}\right)$ and the local voltage drops $\Delta V_{i}\left(t_{k}\right)$ and employing the probability rates $p_{i j}$ we obtain the transfers between nearest neighboring sites. Afterwards the values $\delta_{i}\left(t_{k}\right)$ are updated to a new set of densities $\delta_{i}\left(t_{k+1}\right)$, with which we compute, at time $t_{k+1}$, the local resistivities $\rho_{i}\left(t_{k+1}\right)$, the local voltage drops under the applied voltage $V\left(t_{k+1}\right)$, and finally from Eq.(2) the total resistivity $\rho\left(t_{k+1}\right)$, to start the next simulation step at $t_{k+1}$. Notice that as de VEOV is a $1 \mathrm{D}$ model, the conversion from resistivity to resistance is a trivial scale factor. We refer to the Supp. Information [38] for further details on the numerical values of the parameters employed in the simulations.

As it was already described in Sec.III, the negative forming sets the device in a high resistance state, associated to which we define an initial OV density profile, $\delta_{i}\left(t_{0}\right) \forall i=1 . . N$, to start with the numerical implementation of the VEOV model. This initial OVs profile is chosen to guarantee the post forming high resistance state, in which the $\mathrm{C}$ zone contributes with the dominant resistance, while $\mathrm{L}$ and $\mathrm{R}$ layers present both a lower resistivity due to a large density of OVs, and contribute little to the total resistance (see Figure7 III) (a) for a scketch of the initial post forming state).

Figure 7 panel I) shows the TWL-like HSL obtained with the VEOV model simulations, for a symmetric voltage protocol $V(t)$ following the cycle $0 \rightarrow \mathrm{V}_{m 1}=2.1 \mathrm{~V} \rightarrow-\mathrm{V}_{m 2}=2.1$ $\mathrm{V} \rightarrow 0$. We start from an initial OVs distribution compatible with the post forming state, scketched in panel III) (a). This initial profile gives a resistance of $3 \mathrm{~K} \Omega$, in perfect agreement with the reported experimental value. The positive stimulus moves OVs from the L layer into the C layer (see panel III)(b)), initially tending to reduce the resistance. However, as the applied voltage is increased a strong electric field develops, moving OVs further away to accumulate finally in the $\mathrm{R}$ layer. This gives the RESET transition to the $\mathrm{HR}_{1}$ state displayed in the HSL of panel I). The associated OVs density profile along the active region is shown in the colour map of panel II) whilst the total density of OVs in the L, C and R regions respectively, is sketched in panel III) (c). The $\mathrm{HR}_{1}$ state is mantained in the range $V_{m 1} \rightarrow 0$, until the polarity of the stimulus changes and consequently OVs reverse their motion. For $V=-1.4 \mathrm{~V}$ a SET transition to the $\mathrm{LR}_{1}$ state takes place. The associated OVs distribution is shown in panel II) (row labeled $L_{1}$ ) with the color level in each of the L, C and R regions scketched in panel III)(d), proportional to the total OV density 

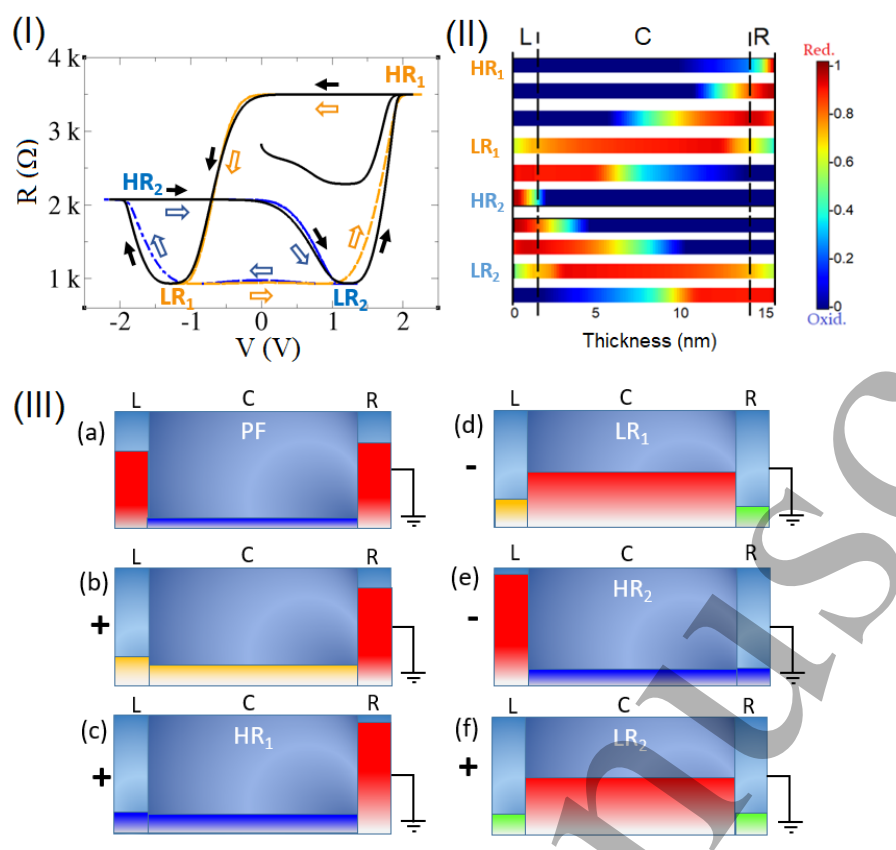

FIG. 7: I) Simulated HSL's. The TWL-like shape is obtained for symmetric stimuli (black arrows indicate the circulation) and squared CW and CCW HSLs for asymmetric ones (blue and orange arrows indicate the circulation in the respective HSLs). The HR and LR states are labeled as described in the text; II) Colormap of the OVs density per site (total density normalized to 1) for different resistance states, as labeled respectively in the HSL of panel I); III) Scheme of the L, C and R regions defined in Figure 6 (b), where the colors qualitatively show the different total number of OVs in each region for: (a) post forming (PF) state, (c)-(f) $\mathrm{HR}_{1}, \mathrm{LR}_{1}, \mathrm{HR}_{2}$ and $\mathrm{LR}_{2}$ states, as labeled in panel I).

respectively. Notice that the R layer is mostly depleted from OVs which accumulate in the $\mathrm{C}$ layer. In spite of this accumulation, zone $\mathrm{C}$ still dominates the device resistance in the low resistance state $L_{1}$. Increasing further the intensity of the negative voltage, produces the electromigration of $\mathrm{OV}$ from the $\mathrm{C}$ to the $\mathrm{L}$ layer, almost voiding of $\mathrm{OVs}$ the $\mathrm{C}$ layer and promoting a second RESET transition to the $\mathrm{HR}_{2}$ state at a voltage $V=-2 \mathrm{~V}$ (see also row labeled $\mathrm{HR}_{2}$ in panel II) and panel III) (e)). The OVs density profile for this case results slightly different from the previous one obtained for the $\mathrm{HR}_{1}$ state, giving $\mathrm{HR}_{2}<$ $\mathrm{HR}_{1}$, in fully agreement with the experimental TWL-like HSL. The $\mathrm{HR}_{2}$ state is mantained until the voltage changes to possitive values and the OVs electromigrate from the $\mathrm{L}$ to the C layer, attaining the $\mathrm{LR}_{2}$ state at $V=1.2 \mathrm{~V}$.

The agreement between the simulated and the experimental TWL-like HSL show in Figure 


\section{DISCUSSION AND CONCLUSIONS}

Our work shows that the symmetry of our devices electrical/response is decoupled from the symmetry of the initial device, the former being controlled by both the electroforming process and the stimuli protocol in the memristive regime. The performed experiments and modeling show that it is possible to selectively activate/deactivate two series memristive interfaces in order to obtain multilevel HSLs with opposite polarities or a "table with legs" on the same device, just by controlling the maximum $\left(V_{\max }>0\right)$ and minimum $\left(V_{\min }<0\right)$ applied voltage excursions. This behaviour is independent of the nature of the used electrodes: we found it in $\mathrm{Pt} / \mathrm{TaO}_{2-h} / \mathrm{Ta}_{2} \mathrm{O}_{5-x} / \mathrm{TaO}_{2-y} / \mathrm{Pt}$ devices, but also in similar systems where the top $\mathrm{Pt}$ electrode was replaced by Au, as reported in Fig. S6 of the Suppl. Information.

A key factor that allows the obtained selective response is a forming process that changes the initial asymmetric Ta-oxide bilayer, grown at different oxygen pressures, to a quasisymmetric $\mathrm{TaO}_{2-h} / \mathrm{Ta}_{2} \mathrm{O}_{5-x} / \mathrm{TaO}_{2-y}$ stack, excluding the $\mathrm{Pt}(\mathrm{Au}) / \mathrm{TaO}_{2-h(y)}$ metal-metal interfaces from contributing to the memristive behavior. The finding of CCW and CW HSLs in a single quasi-symmetric device goes against the common wisdom that, for interface-related memristors, "(a)symmetric systems give (a)symmetric electrical response". We propose, based on our thourough analysis and modeling, that the physical origin of the obtained electrical behayior relies on OVs electromigration at the nanoscale, between a central and interfacial zones of the $\mathrm{Ta}_{2} \mathrm{O}_{5-x}$ layer, sandwiched between metallic $\mathrm{TaO}_{2-h(y)}$. From the combination of experimental results and simulations it can be stated that for CCW HSL the OVs dynamics is constrained to $\mathrm{C}$ and L zones, while for CW HSL the OV exchange is limited between $\mathrm{C}$ and $\mathrm{R}$ layers. For symmetric stimuli all $\mathrm{L}, \mathrm{C}$ and $\mathrm{R}$ are involved and the 
memristive response becomes symmetric (TWL-like HSL).

It can be concluded that OV transfer between one interface (i.e. R) and $\mathrm{C}$ zone does not start until the other interface (i.e. L) is almost completely drained of OV. This 2steps process is the core of the observed behavior. It is worth to remark that this rich phenomenology was found for a system with a rather simple room temperature fabrication process that includes only one oxide-Ta-oxide- and one metal, suggesting that it might be possible to take advantage of these properties at low cost and with easy scability.

Regarding possible applications, the control of the symmetry of the electric response allows optimizing, for example, ON-OFF ratios for RRAM memories; however, the results presented here have potentially more implications for the development of disruptive electronics such as neuromorphic computing or novel logic devices. The observation of multilevel resistance states indicates that our devices can mimic the (analogic) adaptable synaptic weight of biological synapsis. Importantly, the multilevel states were found for both $\mathrm{CCW}$ and CW HSLs, indicating that the synaptic weight could be either potentiated or depressed with electrical stimulus of the same polarity by the application of proper trains of voltage pulses [39]. This might have implications for novel, beyond von Neumann, logic devices.

\section{ACKNOWLEDGEMENTS}

We acknowledge support from INN-CNEA, UNCuyo (06/C455), ANPCyT (PICT20141382, PICT2016-0867, PICT2017-1836, PICT 2017-0984), CONICET (PIP 11220150100653CO) and Univ. Buenos Aires (UBACyT 20020170100284BA). M.A. also acknowledges financial support of H2020-MSCA-RISE-2016 SPICOLOST Grant No. 734187 to perform TEM studies at LMA-INA, University of Zaragoza. S.B. thanks technical support from N. Cortes.

[1] D. Ielmini and R. Waser, Resistive Switching: From Fundamentals of Nanoionic Redox Processes to Memristive Device Applications (Wiley-VCH, 2016).

[2] J. Borghetti, G. S. Snider, P. J. Kuekes, J. J. Yang, D. R. Stewart, and R. S. Williams, Nature 464, 873 (2010).

[3] S. Yu, Neuro-inspiring computing using resistive synaptic devices (Springer International Publishing, 2017). 
[4] A. Prakash, D. Jana, and S. Maikap, Nanoscale Research Letters 8, 418 (2013).

[5] M.-J. Lee, C. B. Lee, D. Lee, S. R. Lee, M. Chang, J. H. Hur, Y.-B. Kim, C.-J. Kim, D. H. Seo, S. Seo, U.-I. Chung, I.-K. Yoo, and K. Kim, Nature Materials 10, 625 (2011).

[6] K. X. Shi, H. Y. Xu, Z. Q. Wang, X. N. Zhao, W. Z. Liu, J. G. Ma, and Y. C. Liu, Appliêd Physics Letters 111, 223505 (2017), https://doi.org/10.1063/1.5002571.

[7] G. H. Baek, A. R. Lee, T. Y. Kim, H. S. Im, and J. P. Hong, Applied Physics Letters 109, 143502 (2016), https://doi.org/10.1063/1.4963884.

[8] H. Zhang, Y. Sijung, S. Menzel, C.-F. W. D. J. H. C. S. W. R. Funck, Carsten, and S. Hoffmann-Eifert, ACS Applied Materials Interfaces 10,29766 (2018).

[9] M. J. Rozenberg, M. J. Sánchez, R. Weht, C. Acha, F. Gomez-Marlasca, and P. Levy, Phys. Rev. B 81, 115101 (2010).

[10] C. La Torre, A. Kindsmller, D. J. Wouters, C. E. Graves, G. A. Gibson, J. P. Strachan, R. S. Williams, R. Waser, and S. Menzel, Nanoscale 9, 14414 (2017).

[11] T. H. Park, S. J. Song, H. J. Kim, S. G. Kim, S. Chung, B. Y. Kim, K. J. Lee, K. M. Kim, B. J. Choi, and C. S. Hwang, Scientific Reports 5, 15965 (2015).

[12] T. H. Park, H. J. Kim, W. Y. Park, S. G. Kim, B. J. Choi, and C. S. Hwang, Nanoscale 9, 6010 (2017).

[13] Y. Yang, P. Sheridan, and W. Lu, Applied Physics Letters 100, 203112 (2012).

[14] N. Ghenzi, M. J. Sánchez, and P. Levy, Journal of Physics D: Applied Physics 46, 415101 (2013).

[15] N. Ghenzi, M. J. Sánchez, D. Rubi, M. J. Rozenberg, C. Urdaniz, M. Weissman, and P. Levy, Applied Physics Letters 104, 183505 (2014).

[16] X. Chen, N. J. Wu, J. Strozier, and A. Ignatiev, Applied Physics Letters 87, 233506 (2005), https://doi.org/10.1063/1.2139843.

[17] F. Nardi, S. Balatti, S. Larentis, and D. Ielmini, 2011 International Electron Devices Meeting, 10.1109/IEDM.2011.6131647 (2011).

[18] S. A. Mojarad, J. P. Goss, K. S. K. Kwa, P. K. Petrov, B. Zou, N. Alford, and A. O’Neill, Journal of Applied Physics 112, 124516 (2012).

[19] S. Kim, S. Choi, and W. Lu, ACS Nano 8, 2369 (2014).

[20] D. S. Jeong, H. Schroeder, and R. Waser, Nanotechnology 20, 375201 (2009). 
[21] R. Simpson, R. G. White, J. F. Watts, and M. A. Baker, Applied Surface Science 405, 79 (2017).

[22] A. Herpers, C. Lenser, C. Park, F. Offi, F. Borgatti, G. Panaccione, S. Menzel, R. Waser, and R. Dittmann, Advanced Materials 26, 2730 (2014).

[23] S. Balatti, S. Larentis, D. Gilmer, and D. Ielmini, Advanced Materials 25, 1474 (2013).

[24] X. Yang and I.-W. Chen, Scientific Reports 2, 744 (2012).

[25] B. Long, Y. Li, S. Mandal, R. Jha, and K. Leedy, Applied Physics Letters 101, 113503 (2012).

[26] H. Okamoto, Journal of Phase Equilibria 22, 515 (2001).

[27] S. P. Garg, N. Krishnamurthy, A. Awasthi, and M. Venkatraman, Journal of Phase Equilibria 17, 63 (1996).

[28] J. J. Yang, M.-X. Zhang, J. P. Strachan, F. Miao, M. D. Pickett, R. D. Kelley, G. MedeirosRibeiro, and R. S. Williams, Applied Physics Letters 97, 232102 (2010).

[29] Z. Wei, Y. Kanzawa, K. Arita, Y. Katoh, K. Kawai, S. Muraoka, S. Mitani, S. Fujii, K. Katayama, M. Iijima, T. Mikawa, T. Ninomiya, R. Miyanaga, Y. Kawashima, K. Tsuji, A. Himeno, T. Okada, R. Azuma, K. Shimakawa, H. Sugaya, T. Takagi, R. Yasuhara, K. Horiba, H. Kumigashira, and M. Oshima, Tech. Dig. - Int. Electron Devices Meet. 293 (2008).

[30] Y. C. Yang, C. Chen, F. Zeng, and F. Pan, Journal of Applied Physics 107, 093701 (2010).

[31] V. Y.-Q. Zhuo, Y. Jiang, M. H. Li, E. K. Chua, Z. Zhang, J. S. Pan, R. Zhao, L. P. Shi, T. C. Chong, and J. Robertson, Applied Physics Letters 102, 062106 (2013).

[32] C. Acha, Journal of Applied Physics 121 (2017), 10.1063/1.4979723.

[33] C. Acha, A. Schulman, M. Boudard, K. Daoudi, and T. Tsuchiya, Applied Physics Letters 109 (2016), 10.1063/1.4955204.

[34] W. Acevedo Román, C. Acha, M. Sanchez, P. Levy, and D. Rubi, Applied Physics Letters 110 (2017), 10.1063/1.4975157.

[35] C. Acha, Journal of Physics D: Applied Physics 44, 345301 (2011).

[36] M. Cerchez, H. Langer, M. El Achhab, T. Heinzel, D. Ostermann, H. Lder, and J. Degenhardt, Applied Physics Letters 103, 033522 (2013).

[37] J. Blasco, N. Ghenzi, J. Su, P. Levy, and E. Miranda, Microelectronics Reliability 55, 1 (2015).

[38] See Supporting Information for details.. 
1

2

3

4

5

6

7

8

9

10

11

12

13

18

19

20

21

22

23

24

25

26

27

28

29

30

31

32

33

34

35

36

37

38

39

40

41

42

43

44

45

46

47

48

49

50

51

52

53

54

55

56

57

58

59

60

[39] A. Schnhals, J. Mohr, D. Wouters, R. Waser, and S. Menzel, IEEE Electron Device Letters 38, 449 (2017).

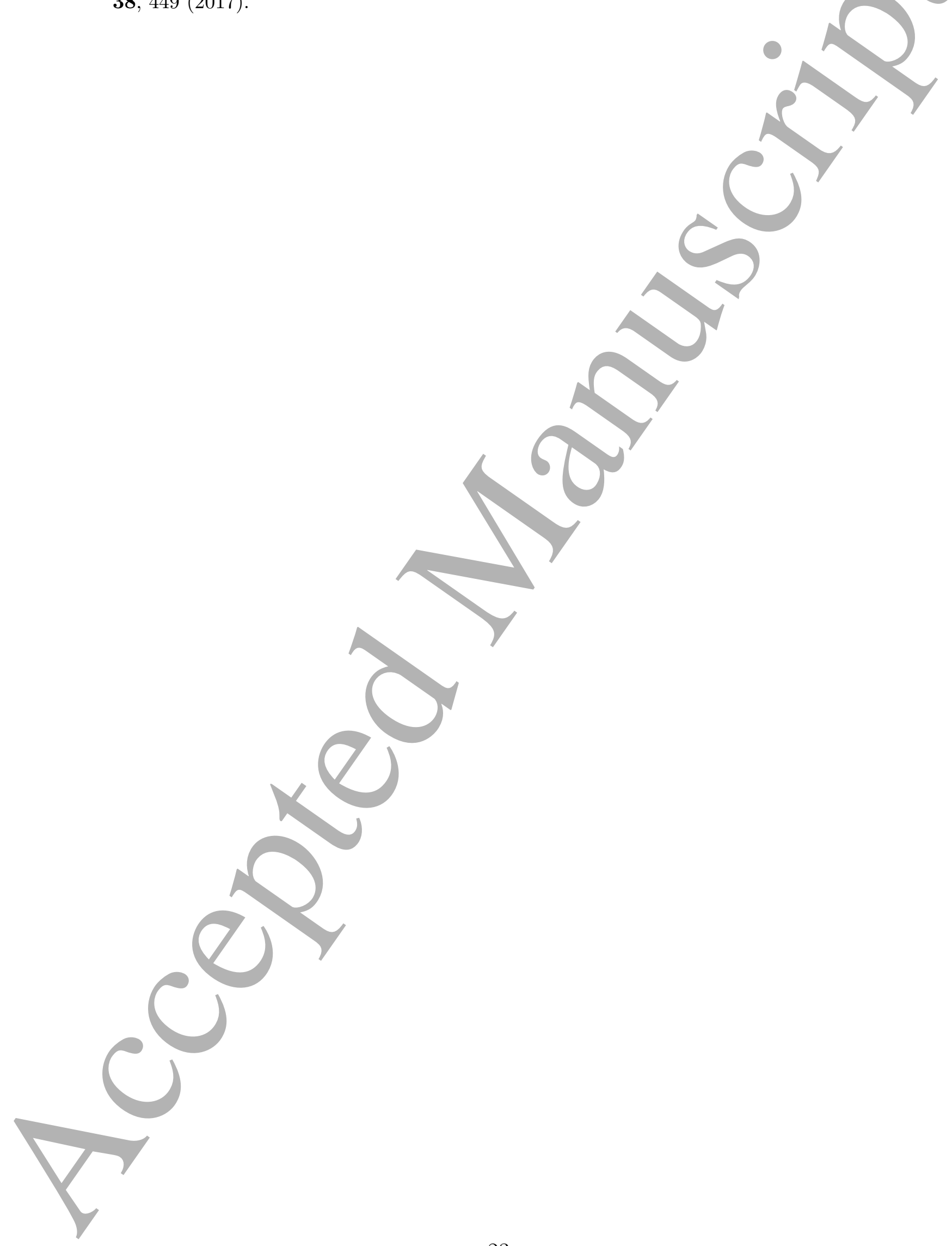

